\title{
Control of Subretinal Fluid: Experimental and Clinical Studies
}

\author{
M. F. MARMOR \\ Stanford, California, USA
}

\begin{abstract}
Summary
Experimental work shows that subretinal fluid is removed both by active transport across the retinal pigment epithelium (RPE) and by passive hydrostatic and oncotic forces that work most effectively when the RPE barrier has been damaged. The retina will stay attached whether or not the RPE is intact-but retinal function requires the RPE barrier and thus active transport is the primary mechanism of subretinal fluid control. RPE fluid transport is normally limited by the retina (which resists water flow from the vitreous) but can be quite powerful when a reservoir of subretinal fluid is present.

Clinical serous detachments are unlikely to form solely as a result of small RPE defects or leaks, since the active and passive transport systems for removing subretinal fluid are both so strong. It is suggested that the primary pathology in most serous retinopathy is a diffuse metabolic or vascular abnormality of RPE fluid transport, and that RPE defects or leaks are necessary but only secondary components of the disease. Several hypotheses for removing subretinal fluid therapeutically are considered in terms of their physiology.

The subretinal space between the photoreceptors and the retinal pigment epithelium (RPE) is the remnant of the embryonic optic vesicle. In the developed eye the subretinal space is of minimal size, but no tissue junctions form across it and it can re-open under pathological conditions of retinal detachment. In a sense, the title of this paper is misleading since normally there should be no subretinal fluid to control. However, ocular mechanisms are necessary to prevent an accumulation of fluid, and to remove it under conditions of stress or disease.
\end{abstract}

\section{Experimental background}

\section{Active water transport across the RPE}

The RPE is a transporting epithelium which moves not only ions and metabolites, but also a net volume of water in an apical-to-basal direction. ${ }^{1,2,3}$ Several 'pumps' and facilitated ion transport systems are involved in water transport, although the specific ionic systems that are most critical in man remain unknown. ${ }^{2,4}$ Our in vivo experiments on rabbits show the importance of metabolic ion transport to fluid movement within the intact eye. If a small amount of saline solution is injected into the subretinal space, it is absorbed within hours. ${ }^{56}$ Absorption is only slightly slower for serum or dextran-containing saline, indicating that ionic fluid can be fully absorbed despite the presence of osmo-

From: The Department of Ophthalmology, Stanford University School of Medicine, Stanford, California. Supported in part by NIH-NEI Research Grant EYO1678 
tically-active large molecules. ${ }^{7,9}$ On the other hand, sucrose solution is not absorbed until ions begin to diffuse into it. ${ }^{6,10}$ The absorption of small experimental detachments is also impeded by generalised metabolic inhibition from anoxia, cyanide or dinitrophenol. .,10,11

Inhibition of the sodium-potassium pump with ouabain, interestingly, appears to enhance the absorption of subretinal fluid. ${ }^{5}$ This makes sense, insofar as the sodium pump is only located on the apical side of the RPE and produces a net movement of sodium into the subretinal space. The carbonic anhydrase inhibitor, acetazolamide, also enhances the absorption of subretinal fluid by a mechanism yet to be determined. ${ }^{5,12}$ The effect has not been duplicated by modifying blood/gas parameters, and appears to involve a direct action of the drug upon the RPE. Rather high doses are required in the rabbit, but lower doses seem useful in primates ${ }^{13}$ and this effect may underlie the recent use of carbonic anhydrase inhibitors to treat cystoid macular oedema. ${ }^{14}$

Subretinal fluid absorption is reduced by exposure to cyclic AMP, ${ }^{15,16}$ which apparently facilitates apical sodium and chloride transport and reduces the net movement of ions out of the eye. ${ }^{17}$ Cyclic GMP has a mild facilitatory effect with both subretinal saline and serum. ${ }^{15,16}$ The involvement of cyclic nucleotides with water transport may explain why adrenalin appears to influence the formation or removal of fluid, in experimental ${ }^{18}$ or clinical serous chorioretinopathy. ${ }^{19}$

The magnitude of fluid movement across the RPE can be quite substantial. By injecting fluid into the subretinal space of rabbits, we estimated the rate of RPE water transport to be $0.12-0.31 \mathrm{ul} / \mathrm{mm}^{2} / \mathrm{hr}^{6,10}$ In vivo studies by others have shown rates of .064 in $\operatorname{dogs}^{3}$ and .073 in monkeys. ${ }^{20}$ These rates would produce bulk flow out of the eye within the same order of magnitude as the flow of aqueous. ${ }^{21}$

Some readers may discern a paradox in this last observation. If the RPE transports fluid at a rate comparable to the secretion of aqueous, how then is intraocular pressure maintained and what is the role for the trabecular meshwork? If the RPE routinely transported fluid at its maximum rate, the inconsistencies would hold, but my guess is that ongoing fluid transport across the normal RPE is actually very small: the rates quoted above represent RPE transport with an unlimited reservoir of fluid, but there is normally little fluid in the subretinal space. The retina provides substantial resistance to fluid flow, ${ }^{22}$ and only a small amount of fluid percolates through in response to intraocular pressure. In other words, the rate-limiting step is ordinarily the passage of fluid through the retina rather than the RPE. When there is a detachment, however, the RPE can transport fluid at its maximal rate (e.g. we know from clinical experience that large amounts of subretinal fluid can absorb within 24 hours after placement of a scleral buckle). ${ }^{23}$

\section{Passive water transport mechanisms}

Although the presence of tight junctions between the RPE cells limits the passive movements of fluid across the RPE, subretinal fluid does respond modestly to hydrostatic or osmotic pressure. Both saline fluid and serum in the subretinal space is absorbed slightly faster when the intraocular pressure is raised from $16 \mathrm{~mm}$ to $38 \mathrm{~mm} \mathrm{Hg}$, and the absorption is prolonged when the pressure is reduced to zero. ${ }^{24}$ Absorption is hastened by intravenous injections of mannitol, which raise the choroidal osmotic pressure ${ }^{25}$ (although this procedure may also alter RPE permeability). ${ }^{26}$ Although an elevated choroidal osmotic pressure may be expected to draw subretinal fluid into the choroid, the mechanisms by which hydrostatic pressure enhances subretinal fluid absorption are less clear. An elevated head of intraocular pressure will drive more fluid per unit time through the semipermeable retina, but this does not easily explain why fluid under the retina should be selectively absorbed. Because of its resistance to flow, the retina may act, under the influence of intraocular pressure, as a tamponade to push out subretinal fluid. ${ }^{27,28}$

\section{Role of the RPE barrier}

There is a tendency for clinicians to view the RPE as a barrier that protects the subretinal space from an influx of choroidal fluid. Experimental evidence, however, indicates exactly the opposite: subretinal fluid is cleared faster than normal when the barrier has been damaged. For example, saline fluid 
injected into the subretinal space will absorb more quickly over a cluster of laser burns than over normal RPE, ${ }^{29}$ and will absorb even faster if the pigment epithelium has been diffusely damaged with sodium iodate. ${ }^{7}$ Presumably, this occurs because the damaged barrier allows choroidal oncotic pressure more easily to draw fluid out of the subretinal space. However, even serum is absorbed effectively over laser burns and iodate-damaged RPE ${ }^{8,29}$ indicating that oncotic pressure is not the only passive driving force. These experimental findings are supported by the clinical results of en bloc removal of choroidal tumours, a procedure that leaves retina overlying bare sclera. ${ }^{30,31}$ Subretinal fluid is rapidly absorbed after this type of surgery, and the retina remains flat despite the total absence of an RPE barrier or RPE transport.

These data tell us that small (or even large) defects in the RPE barrier do not by themselves cause subretinal fluid to accumulate or produce serous detachment. ${ }^{32}$ In fact, the loss of barrier will tend to cause an egress of fluid (a concept that may be part of the effect of photocoagulation in clearing retinal oedema). Is the RPE needed for retinal attachment $?^{33}$ It is not if retinal attachment is all that one requires of the RPE ${ }^{34}$ However, the RPE also controls the metabolic environment of the photoreceptors, phagocytises outer segment debris, and participates in the visual pigment regeneration cycle. Thus, retina may stay attached without the RPE, but it cannot survive functionally. In order to maintain photoreceptor function we require an RPE barrier-and since that barrier blocks the effectiveness of passive fluid transport systems, we must use active transport to move fluid across the RPE.

\section{Clinical implications}

\section{Normal retinal apposition:}

We have seen that the mechanisms of fluid transport across the RPE represent powerful forces to keep the retina in place. In conjunction with other adhesive mechanisms within the subretinal space,$^{28}$ passive forces work to keep the retina apposed, and active transport keeps the subretinal space dehydrated. Clinical detachments are relatively infrequent events that usually (with the exception of a few syndromes such as central serous chorioretinopathy) involve rather severe pathology such as marked choroidal ischaemia or traction from the vitreous. It is hard to understand how a small volume of fluid leaking through a pinpoint RPE defect can overwhelm both the active and passive control mechanisms of the surrounding RPE to produce serous detachment. Some hypotheses about the conditions under which this might occur are considered below. ${ }^{28,32}$

\section{Mechanisms of serous detachment}

The mere presence of a RPE defect or 'leak' is unlikely to be sufficient to create serous detachment. Clearly, a site of fluid entry is necessary, but the experimental and clinical data that have been presented argue strongly that focal defects alone-will not produce detachment (and indeed may enhance fluid removal). Clinical experience tells us that ordinary laser burns do not cause serous detachments. We have found it exceedingly difficult to create serous detachments in animals, even by producing hypotony or choroidal congestion in combination with focal laser burns and cyclic AMP administration. ${ }^{35}$

How, then, does serous fluid form? On theoretical grounds one must have (1) a site of fluid entry; (2) a source of fluid pressure; (3) an inability of the surrounding RPE to remove fluid; and possibly (4) weakened retinal adhesion so that fluid can spread within the subretinal space.

These conditions are met in some drastic pathologic states such as total blockage of choridal venous drainage, but a more subtle balance of factors must be present if we are to explain conditions like central serous chorioretinopathy. When the volume of fluid entering a serous detachment is relatively small, as it must be through a focal RPE defect, then the RPE must be unable (actively or passively) to remove the fluid effectively. This suggests that the underlying RPE barrier is largely intact (or passive fluid egress would occur), but that RPE transport mechanisms are compromised (or the fluid would leave actively). For these reasons, I have proposed $^{32}$ that central serous chorioretinopathy and other serous retinopathies represent diffuse disorders of RPE fluid transport rather 
than disorders of a small leakage site. The leakage site may be necessary, but it is not sufficient, and it may only represent an incidental complication of underlying metabolic or vascular disease involving the RPE and/or choroid.

This concept is consistent with evidence that central serous chorioretinopathy occurs in young men under stress, ${ }^{19}$ and can be mimicked experimentally with chronic adrenalin injections. ${ }^{18}$ It is also consistent with the occurrence of serous detachments in systemic vascular diseases. Laser therapy may hasten the removal of fluid by sealing the immediate leak, ${ }^{8}$ but recurrences will be likely (and occur clinically) unless the underlying metabolic/ vascular abnormality (e.g. the stress response) is relieved. Ultimately to understand the pathogenesis of these diseases, and cure them, our attentions should ideally be focussed on the diffuse RPE transport abnormality rather than the angiographic 'leak'.

\section{Theoretical strategies for enhancing subretinal fluid removal:}

The removal of subretinal fluid could be stimulated, in theory, by either enhancement of active transport systems, enhancement of passive transport forces, or modification of the RPE barrier.

RPE active transport might in theory be enhanced by accelerating general metabolism (e.g. with hyperbaric oxygen) although there is no experimental evidence that this works. Optimising environmental conditions such as blood $\mathrm{pH}$ or temperature might also favour active transport. Evidence has been presented already that acetazolamide ${ }^{5,12}$ and cyclic GMP ${ }^{15,16}$ do facilitate transport, and acetazolamide is in fact being used for the treatment of cystoid macular oedema. ${ }^{14}$ There is dispute whether acetazolamide acts primarily on the pigment epithelium or on the retinal capillaries, ${ }^{36}$ and the drug has not been proven to be of any value in central serous retinopathy. However, carbonic anhydrase inhibitors may eventually have a role in hastening the absorption of subretinal fluid or preventing the development or spread of retinal detachment.

Passive forces can also be modified to alter subretinal fluid movement. Choroidal congestion and serous detachment are complications of severe hypotony; conversely, the cautious elevation of intraocular pressure might be useful in treating certain types of subretinal fluid accumulation. Intravenous mannitol ${ }^{25}$ or similar osmotic agents should help to draw out subretinal fluid, and could be valuable clinically under conditions where the dehydration of the subretinal space would be maintained after the fluid load was removed. The amount of fluid entering the subretinal space from the vitreous is normally very small, so that once a large subretinal fluid load has been dissipated, even a compromised RPE may be able to maintain retinal apposition.

The role of barrier damage in causing or treating detachment is complex. The RPE is obviously damaged by photocoagulation, which does not ordinarily cause detachments, but laser burns also destroy the choriocapillaris which might be needed as a source or sink of fluid. Photocoagulation effects are generally transient, insofar as new RPE cells will cover a laser burn scar within 10 to 14 days. ${ }^{8}$ Macular photocoagulation grids are used clinically to remove diffuse retinal oedema ${ }^{37}$ (which is different, of course, from subretinal fluid), but the beneficial effects may not appear for weeks or months which suggests that the mechanism is not merely an opening of passive egress channels. Nevertheless, there may be situations in which a transient opening of passive RPE 'pores' would be of clinical value, especially if laser or other techniques can be evolved to damage the RPE barrier without destroying the choricapillaris.

\section{References}

${ }^{1}$ Frambach DA, Weiter JJ, Adler AJ: A photogrammetric method to measure fluid movement across isolated frog retinal pigment epithelium. Invest Ophthalmol Vis Sci 1985, 47: 547-52.

${ }^{2}$ Hughes BA, Miller SS, Machen TE: Effects of cyclic AMP on fluid absorption and ion transport across frog retinal pigment epithelium. J Gen Physiol 1984, 83: 875-99.

${ }^{3}$ Tsuboi S: Measurement of the volume flow and hydraulic conductivity across the isolated dog retinal pigment epithelium. Invest Ophthalmol Vis Sci 1987, 28: 1776-82. 
${ }^{4}$ Tsuboi S: Aspects of electrolyte transport across isolated dog retinal pigment epithelium. Am J Physiol 250: (Renal Fluid Electrolyte Physiol 19) 1986, F781-84.

${ }^{5}$ Marmor MF, Abdul-Rahim AS, Cohen DS: The effect of metabolic inhibitors on retinal adhesion and subretinal fluid resorption. Invest Ophthalmol Vis Sci 1980, 19: 893-903.

${ }^{6}$ Frambach DA and Marmor MF: The rate and route of fluid resorption from the subretinal space of the rabbit. Invest Ophthalmol Vis Sci 1982, 22: 292-302.

${ }^{7}$ Negi A and Marmor MF: The resorption of subre- tinal fluid after diffuse damage to the retinal pigment epithelium. Invest Ophthalmol Vis Sci 1983, 24: 1475-79.

${ }^{8}$ Negi A and Marmor MF: Healing of photocoagulation lesions affects the rate of subretinal fluid resorption. Ophthalmology 1984, 91: 1678-83.

${ }^{9}$ Marmor MF, Negi A, Maurice DM: Kinetics of macromolecules injected into the subretinal space. Exp Eye Res 1985, 40: 687-96.

${ }^{10}$ Negi A and Marmor MF: Quantitative estimation of metabolic transport of subretinal fluid. Invest Ophthalmol Vis Sci 1986, 27: 1564-68.

${ }^{11}$ Negi A and Marmor MF: Mechanisms of subretinal fluid resorption in the cat eye. Invest Ophthalmol Vis Sci 1986, 27; 1560-63.

${ }^{12}$ Marmor MF and Maack T: Enhancement of retinal adhesion and subretinal fluid resorption by acetazolamide. Invest Ophthalmol Vis Sci 1982, 23: $121-4$.

${ }^{13}$ Tsuboi S and Pederson JE: Experimental retinal detachment. X. Effect of acetazolamide on vitreous fluorescein disappearance. Arch Ophthalmol 1985, 103: 1557-8.

${ }^{14}$ Cox SN, Hay E, Bird AC: Treatment of chronic macular edema with acetazolamide. Arch Ophthalmol 1988, 106: 1190-95.

${ }^{15}$ Marmor MF and Negi A: Pharmacologic modification of subretinal fluid resorption. Arch Ophthalmol 1986, 104: 1674-77.

${ }^{16}$ Kawano S-I and Marmor MF: Metabolic influences on the absorption of serous subretinal fluid. Invest Ophthalmol Vis Sci 1988, 29: 1255-57.

${ }^{17}$ Hughes BA, Miller SS, Machen TE: Effects of cyclic AMP on fluid absorption and ion transport across frog retinal pigment epithelium. J Gen Physiol 1984, 83: 875-99.

${ }^{18}$ Yoshioka H, Katsume Y, Akune H. Experimental central serous chorioretinopathy in monkey eyes: Fluorescein angiographic findings. Ophthalmologica 1982, 185: 168-78.

${ }^{19}$ Yannuzzi LA: Type A behavior and central serous chorioretinopathy. Retina 1987, 7: 111-30.

${ }^{20}$ Tsuboi S and Pederson JE: Volume flow across the isolated retinal pigment epithelium of cynomolgus monkey eyes. Invest Ophthalmol Vis Sci 1988, 29: $1652-55$.
${ }^{21}$ Johnson F and Maurice D: A simple method of measuring aqueous humor flow with intravitreal fluoresceinated dextrans. Exp Eye Res 1984, 39: 791-805.

${ }^{22}$ Fatt I and Shantinath K: Flow conductivity of retina and its role in retinal adhesion. Exp Eye Res 1971, 12: $218-26$

${ }^{23}$ Lincoff $\mathrm{H}$ and Kreissig I: The treatment of retinal detachment without drainage of subretinal fluid (modifications of the Custodis procedure: Part IV). Trans Am Acad Ophthalmol Otol 1972, 76: 1221-33.

${ }^{24}$ Negi A, Kawano S-I, Marmor MF: Effects of intraocular pressure and other factors on subretinal fluid resorption. Invest Ophthalmol Vis Sci 1987, 28: 2099-102.

${ }^{25}$ Negi A and Marmor MF: Effects of subretinal and systemic osmolality on the rate of subretinal fluid resorption. Invest Ophthalmol Vis Sci 1984, 25: 616-20.

${ }^{26}$ Laties AM and Rapoport S: The blood-ocular barriers under osmotic stress. Arch Ophthalmol 1976, 94: 1086-91.

${ }^{27}$ Foulds WS: The vitreous in retinal detachment. Trans Ophthalmol Soc UK 1975, 95: 412-16.

${ }^{28}$ Marmor MF: Mechanisms of normal retinal adhesion. In Ryan SJ, Schachat AP, Murphy RB, Patz A eds, Retina, St. Louis: CV Mosby Co 1989: 71-87.

${ }^{29}$ Negi A and Marmor MF: Experimental serous retinal detachment and focal pigment epithelial damage. Arch Ophthalmol, 1984, 102: 445-49.

${ }^{30}$ Foulds WS: Experience of local excision of uveal melanomas. Trans Ophthalmol Soc UK 1977, 97 : 412-15.

${ }^{31}$ Peyman GA and Raichand M: Full-thickness eye wall resection of choroidal neoplasms. Ophthalmology 1979, 86: 1024-36.

${ }^{32}$ Marmor MF: New hypotheses on the pathogenesis and treatment of serous retinal detachment. Graefe's Arch Klin Exp Ophthalmol 1988, 226: 548-52.

${ }^{33}$ Foulds WS: Do we need a retinal pigment epithelium (or choroid) for the maintenance of retinal apposition? Br J Ophthalmol 1985, 69: 237-39.

${ }^{34}$ Marmor MF: Commentary on 'Do we need a pigment retinal epithelium (or choroid) for maintenance of retinal apposition?' by W Foulds. Surv Ophthalmol 1986, 34: 344-45.

${ }^{35}$ Tsukahara Y, Kawano S-I, Marmor MF: Studies on the mechanism of serous detachment. Graefe Arch Klin Exp Ophthalmol (Submitted).

${ }^{36}$ Fishman GA, Gilbert LD, Fiscella RG, Kimura AE: Acetazolamide for treatment of chronic edema in retinitispigmentosa. Arch Ophthalmol 1989, 107: 1445-52.

${ }^{37}$ Olk RJ: Modified grid argon (blue-green) laser photocoagulation for diffuse diabetic macular edema. Ophthalmology 1986, 93: 938-50. 